\title{
Measuring the Outreach Efforts of Public Health Authorities and the Public Response on Facebook During the COVID-19 Pandemic in Early 2020: Cross-Country Comparison
}

Aravind Sesagiri Raamkumar ${ }^{1}$, BTech, MSc, PhD; Soon Guan Tan ${ }^{1}$, BSc; Hwee Lin Wee ${ }^{1,2}$, BSc, PhD

${ }^{1}$ Saw Swee Hock School of Public Health, National University of Singapore, Singapore, Singapore

${ }^{2}$ Department of Pharmacy, National University of Singapore, Singapore, Singapore

Corresponding Author:

Hwee Lin Wee, BSc, PhD

Saw Swee Hock School of Public Health

National University of Singapore

MD1 \#10-01

12 Science Drive 2, National University of Singapore

Singapore, 117549

Singapore

Phone: 6565164975

Email: ephwhl@nus.edu.sg

\section{Abstract}

Background: The coronavirus disease (COVID-19) pandemic presents one of the most challenging global crises at the dawn of a new decade. Public health authorities (PHAs) are increasingly adopting the use of social media such as Facebook to rapidly communicate and disseminate pandemic response measures to the public. Understanding of communication strategies across different PHAs and examining the public response on the social media landscapes can help improve practices for disseminating information to the public.

Objective: This study aims to examine COVID-19-related outreach efforts of PHAs in Singapore, the United States, and England, and the corresponding public response to these outreach efforts on Facebook.

Methods: Posts and comments from the Facebook pages of the Ministry of Health (MOH) in Singapore, the Centers for Disease Control and Prevention (CDC) in the United States, and Public Health England (PHE) in England were extracted from January 1, 2019, to March 18, 2020. Posts published before January 1, 2020, were categorized as pre-COVID-19, while the remaining posts were categorized as peri-COVID-19 posts. COVID-19-related posts were identified and classified into themes. Metrics used for measuring outreach and engagement were frequency, mean posts per day (PPD), mean reactions per post, mean shares per post, and mean comments per post. Responses to the COVID-19 posts were measured using frequency, mean sentiment polarity, positive to negative sentiments ratio (PNSR), and positive to negative emotions ratio (PNER). Toxicity in comments were identified and analyzed using frequency, mean likes per toxic comment, and mean replies per toxic comment. Trend analysis was performed to examine how the metrics varied with key events such as when COVID-19 was declared a pandemic.

Results: The MOH published more COVID-19 posts ( $\mathrm{n}=271$; mean PPD 5.0) compared to the CDC ( $\mathrm{n}=94$; mean PPD 2.2) and PHE ( $\mathrm{n}=45$; mean PPD 1.4). The mean number of comments per COVID-19 post was highest for the CDC (mean CPP 255.3) compared to the MOH (mean CPP 15.6) and PHE (mean CPP 12.5). Six major themes were identified, with posts about prevention and safety measures and situation updates being prevalent across the three PHAs. The themes of the MOH's posts were diverse, while the CDC and PHE posts focused on a few themes. Overall, response sentiments for the MOH posts (PNSR 0.94) were more favorable compared to response sentiments for the CDC (PNSR 0.57) and PHE (PNSR 0.55) posts. Toxic comments were rare $(0.01 \%)$ across all PHAs.

Conclusions: PHAs' extent of Facebook use for outreach purposes during the COVID-19 pandemic varied among the three PHAs, highlighting the strategies and approaches that other PHAs can potentially adopt. Our study showed that social media analysis was capable of providing insights about the communication strategies of PHAs during disease outbreaks.

(J Med Internet Res 2020;22(5):e19334) doi: 10.2196/19334 


\section{KEYWORDS}

COVID-19; sentiment analysis; emotion analysis; public health authorities; infectious disease; outbreak; public engagement; social media; public health; virus

\section{Introduction}

\section{Background}

The coronavirus disease (COVID-19) was first identified in Wuhan, China in December 2019. It has since spread to 210 countries and territories, infecting 1,697,356 people and causing 102,667 deaths as of April 11, 2020 [1]. Compared to the previous outbreaks of severe acute respiratory syndrome (SARS) and Middle East respiratory syndrome, COVID-19 has caused more infections and deaths, spreading from an infected person to 2-2.5 people on average [2]. Most countries started reporting infections by the second half of January 2020. The United States reported its first case on January 20, 2020 [3], while England, under the United Kingdom, reported its first cases on January 31, 2020 [4]. Singapore reported its first case on January 23, 2020 [5]. In an effort to contain the COVID-19 pandemic in Singapore, multiple interventions have been implemented on both societal and health care system levels [6], and the country shifted rapidly to Disease Outbreak Response System Condition (DORSCON) orange, the second-highest level of alert for disease outbreaks in Singapore, on February 7, 2020 [7], just 15 days after the first case of COVID-19 infection was confirmed. The World Health Organization (WHO) declared this disease as a pandemic on March 11,2020, and is unable to ascertain the duration of the pandemic [8].

Countries such as Singapore, Taiwan, and South Korea have taken the necessary precautions to handle this pandemic within their borders in January when the pandemic was largely confined to China [9]. On the international scene, Singapore has received accolades from the WHO and several world leaders praising our efforts in containing the disease [10,11]. The Dean of the Saw Swee Hock School of Public Health, National University of Singapore, has also been invited by several overseas universities to share Singapore's experience in combating the COVID-19 pandemic $[12,13]$. Unfortunately, precautionary measures have been reported to be found wanting in other countries such as the United States and the United Kingdom $[14,15]$. It is to be noted that such delays in preparation for epidemics have also been seen in the past with Zika, influenza A virus subtype H1N1, and Ebola [16].

\section{Social Media Use During Pandemics}

Effective risk communication is essential in directing the public to adopt certain desired behaviors such as social distancing and good hygiene habits in times of pandemics. Transparent and consistent communication amidst the uncertainty of the pandemic is also crucial in maintaining public confidence and trust $[17,18]$. Traditionally, the government and public health authorities (PHAs) relied on websites, news media, print press, and television as main platforms for the dissemination of pandemic-related news and information to the public. In contrast to the 2003 SARS and 2009 H1N1 pandemic, present-day media landscapes worldwide have evolved significantly, with a greater presence of social media and alternative local and overseas media outlets [19]. The advent of social media platforms such as Facebook and Twitter facilitated the instantaneous sharing of information during pandemics for both the health authorities and the general public. With widespread social media use and the participatory web, PHAs must understand that health risk communication is no longer a linear process [20]. The public can voice their sentiments and comments on the actions undertaken by the government as events related to the pandemic unfold. The public themselves are also involved in content creation through blogs and forums. Citizen journalism and propagation of information pertaining to a pandemic is made possible within their social networks.

Existing research on social media has explored epidemics and pandemics such as Zika [21-23], H1N1 [24], and Ebola [25]. The scope of these studies includes descriptive analysis of posting frequency [21], thematic analysis of post content [26], sentiment analysis of posts [23], and social network analysis [24]. Although the WHO has put forth guidelines for emergency risk communication during epidemics [27], countries may adopt different strategies when conveying health risks across social media platforms. Currently, there is a lack of studies that compare the social media outreach efforts of PHAs from different countries and corresponding responses and interactions by the general public. Such studies might offer rich insights on how effectively platforms such as Facebook could be used for risk communication.

\section{This Study}

Amid the uncertainty of a health threat such as COVID-19, the public have a greater demand for real time, transparent, and consistent messaging. Government agencies run the risk of losing the centralized control of the risk communication process if they do not act swiftly to public sentiment and dispel falsehoods and misinformation [18,28]. A confluence of factors could lead to unintended behavioral outcomes among the public in the ongoing COVID-19 pandemic. The mismatch in perceived threats as well as costs and benefits of certain health behaviors communicated by either mainstream media, government authorities, or alternative media could result in a distorted understanding among the general public. Hence, it is crucial to understand how the prevailing sentiments and narratives about the pandemic were conveyed through the different communication channels and how it was received by the general public who have access to these channels. This will highlight the trigger points, allowing health authorities to fine-tune messaging along the course of the pandemic to allay public fear and panic.

Hence, in this study, we seek to answer four research questions related to Facebook use during a pandemic. First, how frequently do the PHAs of Singapore, the United States, and England use Facebook for risk communication? Second, what were the primary themes of the COVID-19-related posts by PHAs? Third, what are the Facebook followers' sentiments and emotions in response to these COVID-19-related posts by PHAs? Fourth, 
how common are toxic comments that may incite public unrest, and do these toxic comments gain traction? We have selected Singapore, the United States, and England for this study, as we intend to look at the findings from a cross-country perspective and these are developed countries that have English as their official language.

\section{Methods}

\section{Data Extraction}

Data for this study were extracted from three Facebook pages using the tool Facepager [29] on March 19, 2020. The three Facebook pages are officially managed by the Ministry of Health $(\mathrm{MOH})$, Singapore [30], the Centers for Disease Control and Prevention (CDC) in the United States [31], and Public Health England (PHE) in England [32]. As of April 4, 2020, the followers count of the $\mathrm{MOH}$, the CDC, and PHE are 212,453, $2,636,072$, and 336,935, respectively. Extracted data include posts by PHAs, comments from Facebook users, and their corresponding reactions, a feature in Facebook where users can interact with a Facebook status update, article, or a photo or video using one of six emotional reactions: Like, Love, Haha, Wow, Sad, and Angry. Contents posted between January 1, 2019, and March 18, 2020, were analyzed. Posts before January 1, 2020, were considered pre-COVID-19, and posts after January 1, 2020, were considered peri-COVID-19. We selected January 1,2020 , as the starting point for peri-COVID-19 since the statement on "Precautionary Measures in Response to Severe Pneumonia Cases in Wuhan, China," was issued by the $\mathrm{MOH}$ on January 2, 2020 [33]. COVID-19-related posts were filtered out by manually scanning through the textual content of the posts.

\section{Data Analysis}

\section{Extent of Facebook Use}

To examine the extent of Facebook use by the MOH, the CDC, and PHE, we calculated the average number of daily posts and compared pre-COVID-19 and peri-COVID-19 phases. To identify how specific events may influence the extent of Facebook use, we related the number of daily posts to the key dates on which the three countries reported their first COVID-19 cases or declared the outbreak as a national-level pandemic through the average number of posts per day (PPD) measure. To evaluate the extent of public engagement with the Facebook posts, we calculated the average number of reactions per post (RPP), average number of shares per post (SPP), and average number of comments per post (CPP) for pre- and peri-COVID-19 periods but focusing on COVID-19-related posts for the peri-COVID-19 period.

\section{Thematic Analysis of COVID-19 Posts}

The prevalent theme of each COVID-19 post was identified using a process involving two coders. First, the principal coder reviewed the contents of the COVID-19 posts by PHAs and assigned the relevant themes [34]. Next, another coder reviewed and confirmed the themes assigned by the first coder. The content of the posts were first screened through and condensed into short units. The predominant theme conveyed in the post (ie, the theme taking the larger proportion of the message) was assigned to posts with more than one theme. The list of themes included situation update, preventive measures, appreciation, public reassurance, disease information, falsehood correction, face mask, research, testing and diagnosis, and miscellaneous. For each of these themes, the number of constituent posts and percentage of these posts to the total number of posts were reported.

\section{Sentiment and Emotion Analyses of Comments to COVID-19 Posts}

To gain insights into the reactions and comments by Facebook users on the posts by PHAs, we conducted sentiment and emotion analyses on comments written in the English language. The sentiment polarity (SP) score for each comment was identified using the Vader algorithm [35] since the algorithm has been specifically conceptualized for ascertaining the sentiment in short texts (eg, user comments on Facebook or tweets from Twitter). The SP score value ranges from -1 to 1 and was classified into five categories: verynegative $(-1<$ score $<-0.5)$, negative $(-0.5<$ score $<0)$, neutral $($ score $=0)$, positive $(0<$ score $<0.5)$, or very positive $(0.5<$ score $<1)$.

For emotion analysis, we went beyond the emotions available as Facebook reactions. We adopted the eight emotions put forth in the theory of emotion [36] and classified the emotions of the comments as: anger, fear, sadness, disgust, surprise, anticipation, trust, or joy. Among the eight emotions, trust and joy are positive emotions, while anger, sadness, fear, and disgust are considered negative emotions. Surprise and anticipation can be either positive or negative depending on the context, hence not included in either of the two categories. The emotions conveyed in the comments were identified with the help of the DeepMoji algorithm [37] using a two-step process. First, the comments were analyzed using the DeepMoji algorithm, which recommended emojis based on the textual content. Second, the emotion that was mapped to the first-ranked emoji was considered as the emotion for the comment. The table in Multimedia Appendix 1 lists the mapping between the emojis and the corresponding emotions.

The total number of comments, CPP, SP scores, positive to negative sentiments ratio (PNSR) and positive to negative emotions ratio (PNER) were reported. PNSR and PNER are two valid measures that have been used in prior studies for sentiment and emotion analysis in texts [38-40]. The number of comments and the SP scores were plotted alongside the dates of key events.

\section{Identification of Toxicity in Comments of COVID-19 Posts}

Besides the general sentiment and emotion analyses, we identified toxic comments that may warrant intervention. Toxicity is defined as "the usage of rude, disrespectful, or unreasonable language that will likely provoke or make another user leave a discussion" [41]. In this study, the Perspective application program interface (API) service of Google [42] was used to measure the toxicity of comments. The toxicity score ranges between 0 (nontoxicity) and 1 (full toxicity). We further categorized the comments into a dichotomous variable, where comments with a toxicity score greater than or equal to 0.75 are 
toxic and comments with toxicity scores less than 0.75 are nontoxic. We examined the number of likes and replies per toxic comment to determine if such toxic comments may have any ripple effect. We compared this to the number of likes and replies per nontoxic comment as a reference.

\section{Results}

\section{COVID-19 Outreach Efforts of PHAs and Public Engagement}

In Table 1 and 2, the aggregated statistics related to the Facebook posts from the $\mathrm{MOH}$, the CDC, and PHE are listed along with the public engagement metrics. In Figure 1, the COVID-19 posts daily count values are plotted in a line graph. In addition, the key dates on which the three countries reported their first COVID-19 cases and declared the outbreak as a national-level pandemic (in national terminology) are highlighted in the figure.

In the pre-COVID-19 phase (January 1, 2019, to December 31, 2019), the CDC had an average of 2 daily posts, while the $\mathrm{MOH}$ and PHE had an average of 1 daily post (Table 1). The $\mathrm{MOH}$ stepped up on the use of Facebook for public engagement during COVID-19. As of March 18, 2020, the MOH had published 304 posts peri-COVID-19 (January 1, 2020, to March 18, 2020), which was nearly a threefold increase in their PPD from 1.35 to 4.34. The MOH had the highest frequency of postings among the three PHAs with 304 posts, and the majority were related to COVID-19. The mean number of daily posts was 4.3, ranging from 1 to 15 posts daily. February was the most active month with 132 COVID-19 posts. Prior to the first locally confirmed case in Singapore on January 23, 2020, there was a limited number of daily posts from the $\mathrm{MOH}$ (ranging from 1 to 3 daily posts). However, the number of daily posts from the $\mathrm{MOH}$ increased to 7 two days after the first confirmed case in Singapore, and there have been at least 2 PPD ever since.

Of the total CDC posts, less than half were related to COVID-19. It published the highest number of COVID-19 posts in March, with 53 posts in the first 18 days of the month. The highest number of posts published on a single day was on March 8 , 2020 , with 5 posts. The CDC started to post at least 1 COVID-19 post every day starting on February 25, 2020. The number of daily posts remained at low levels even after the United States had declared a level 1 emergency on January 30, 2020. The average number of daily posts increased only after the United States declared COVID-19 as a level 1 emergency on January 30,2020 , and rose again after the WHO declared a pandemic on March 11, 2020.

PHE had the lowest posting frequency with only about half of posts being related to COVID-19 posts. Similar to the $\mathrm{MOH}$, February was the most active month with 19 COVID-19 posts for PHE, and the highest number of posts on a single day $(n=3)$ was done on 3 days: January 24, March 4, and March 13, 2020. Despite the United Kingdom declaring COVID-19 as a level 4 incident on March 3, 2020, there were days when PHE had zero COVID-19 posts. PHE started posting at least one COVID-19 post every day on March 13, 2020.

Compared to the pre-COVID-19 phase (Table 2), there was also a considerable increase in the public engagement metrics in the peri-COVID-19 time period. For instance, the MOH had a 7-fold increase in CPP, with a higher average number of people commenting in 2020 compared to 2019. The CDC saw a 9-fold increase in the mean RPP from 2019 to 2020 and close to a 10 -fold increase in mean SPP from 2019 to 2020. In the case of PHE, the biggest rise is seen for SPP, with nearly a 5-fold increase from 2019 to 2020.

We have also noted that Facebook users who read peri-COVID-19 posts from the MOH were more likely to react to the post than to share or comment on the post, while Facebook users who read posts from the CDC and PHE were more likely to share the posts than to react or comment on the post. This observation was consistent in both pre- and peri-COVID-19 periods.

Table 1. Summary of COVID-19 Facebook outreach by the MOH, the CDC, and PHE during the periods of pre-COVID-19 (January 1, 2019 , to December 31, 2019) and peri-COVID-19 (January 1, 2020, to March 18, 2020).

\begin{tabular}{lllllll}
\hline Agency & $\begin{array}{l}\text { Outreach effort (pre-COVID-19 } \\
\text { Total posts, } \mathrm{n}\end{array}$ & PPD $^{\mathrm{b}}$, mean (SD) & Total posts, $\mathrm{n}$ & PPD, mean (SD) & $\begin{array}{l}\text { COVID-19 posts, } \\
\mathrm{n}(\%)\end{array}$ & $\begin{array}{l}\text { COVID-19 PPD Since first } \\
\text { reported case in each coun- } \\
\text { try, mean (SD) }\end{array}$ \\
\hline $\mathrm{MOH}^{\mathrm{c}}$ & 192 & $1.4(1.09)$ & 304 & $4.3(3.5)$ & $271(89.1)$ & $5.0(3.6)$ \\
$\mathrm{CDC}^{\mathrm{d}}$ & 599 & $2.1(0.99)$ & 232 & $2.1(1.1)$ & $94(40.5)$ & $2.2(1.1)$ \\
$\mathrm{PHE}^{\mathrm{e}}$ & 346 & $1.3(0.56)$ & 87 & $1.6(0.8)$ & $45(51.7)$ & $1.4(0.7)$ \\
\hline
\end{tabular}

${ }^{\mathrm{a} C O V I D-19:}$ coronavirus disease.

${ }^{\mathrm{b}} \mathrm{PPD}$ : posts per day.

${ }^{\mathrm{c}} \mathrm{MOH}$ : Ministry of Health.

${ }^{\mathrm{d}} \mathrm{CDC}$ : Centers for Disease Control and Prevention.

${ }^{\mathrm{e}}$ PHE: Public Health England. 
Table 2. Summary of COVID-19 Facebook engagement by the MOH, the CDC, and PHE during the periods of pre-COVID-19 (January 1, 2019 , to December 31, 2019) and peri-COVID-19 (January 1, 2020, to March 18, 2020).

\begin{tabular}{|c|c|c|c|c|c|c|}
\hline \multirow[t]{2}{*}{ Agency } & \multicolumn{3}{|c|}{ Public engagement (pre-COVID-19 ${ }^{\mathrm{a}}$ ) } & \multicolumn{3}{|c|}{ Public engagement (peri-COVID-19) } \\
\hline & $\mathrm{RPP}^{\mathrm{b}}$, mean $(\mathrm{SD})$ & $\mathrm{SPP}^{\mathrm{c}}$, mean $(\mathrm{SD})$ & $\mathrm{CPP}^{\mathrm{d}}$, mean $(\mathrm{SD})$ & $\mathrm{RPP} \_\mathrm{C} 19^{\mathrm{e}}$, mean $(\mathrm{SD})$ & SPP_C19 ${ }^{\mathrm{f}}$, mean $(\mathrm{SD})$ & CPP_C19 ${ }^{g}$, mean $(\mathrm{SD})$ \\
\hline $\mathrm{MOH}^{\mathrm{h}}$ & $34.3(26.3)$ & $20.1(100.3)$ & $2.2(4.5)$ & $188.9(201.4)$ & $84.6(279.7)$ & $15.6(20.7)$ \\
\hline $\mathrm{CDC}^{\mathrm{i}}$ & $230.7(203.4)$ & $240.9(697.8)$ & $43.1(72.7)$ & $2128.2(4864.9)$ & $2373.8(3485.8)$ & $255.3(298.3)$ \\
\hline $\mathrm{PHE}^{\mathrm{j}}$ & $52.4(68.7)$ & $102.6(212.5)$ & $4.3(6.7)$ & $101.5(89.8)$ & $478.9(568.1)$ & $12.5(11.5)$ \\
\hline
\end{tabular}

${ }^{\mathrm{a} C O V I D-19:}$ coronavirus disease.

${ }^{\mathrm{b}} \mathrm{RPP}$ : reactions per post.

${ }^{\mathrm{c}} \mathrm{SPP}$ : shares per post.

${ }^{\mathrm{d}} \mathrm{CPP}$ : comments per post.

${ }^{\mathrm{e}} \mathrm{RPP}$ C19: reactions to COVID-19 post.

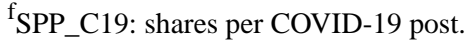

${ }^{\mathrm{g}}$ CPP_C19: comments per COVID-19 post.

${ }^{\mathrm{MOH}}$ : Ministry of Health.

${ }^{\mathrm{i}} \mathrm{CDC}$ : Centers for Disease Control and Prevention.

${ }^{\mathrm{j}}$ PHE: Public Health England.

Figure 1. COVID-19 posts frequency during the analysis period. CDC_PC: Centers for Disease Control and Prevention's number of posts; COVID-19: coronavirus disease; DORSCON: Disease Outbreak Response System Condition; MOH_PC: Ministry of Health's number of posts; PHE_PC: Public Health England number of posts; SG: Singapore; WHO: World Health Organization.

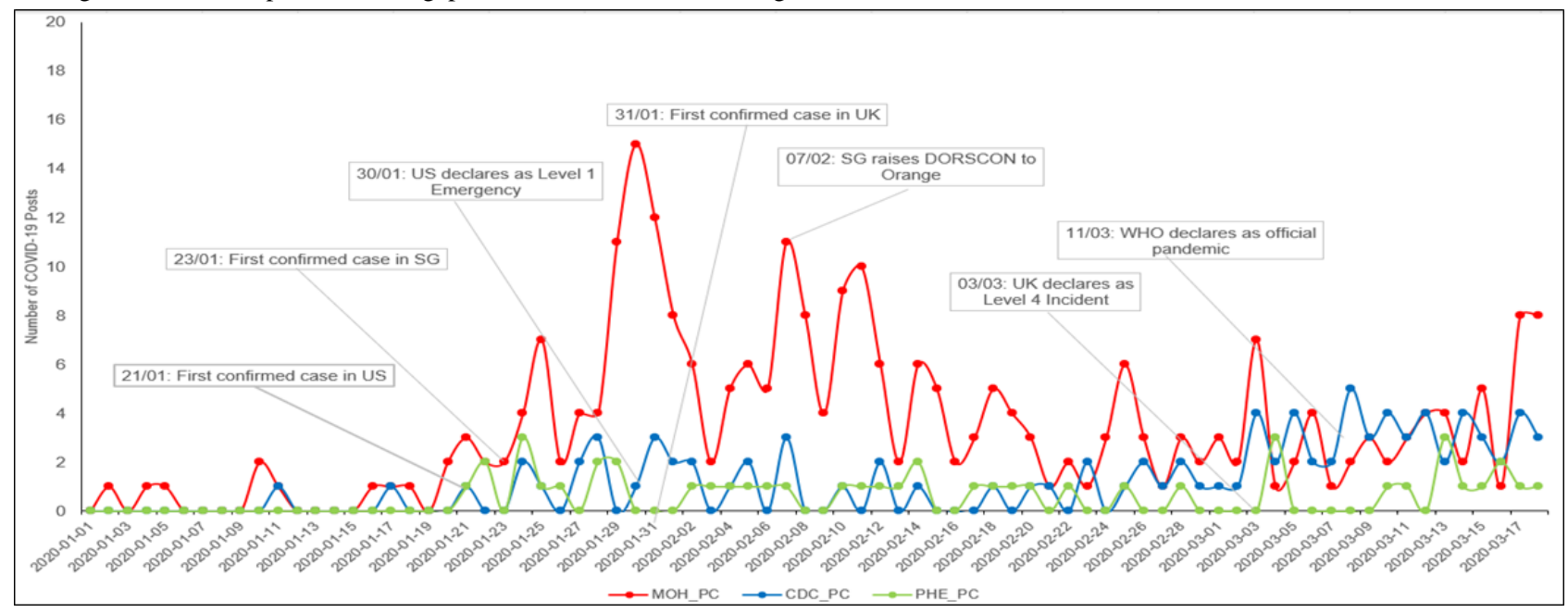

\section{Thematic Analysis}

The themes appreciation, research, testing and diagnosis, and miscellaneous were combined to the theme others to focus on six major themes. As shown in Table 3, the themes from the $\mathrm{MOH}$ are more diverse, with no theme exceeding $30 \%$ of the total posts. In contrast, the CDC and PHE posts were largely related to preventive measures. For the $\mathrm{MOH}$, situation update and preventive measures were the top two themes. Interestingly, the CDC and PHE did not issue any post to correct false information, while the $\mathrm{MOH}$ issued 16 of such posts. There was also no post from PHE and only 2 posts from the CDC to reassure their Facebook followers, while the MOH issued 32 of such public reassurance posts. 
Table 3. Thematic analysis of the public health authorities' coronavirus disease outreach efforts.

\begin{tabular}{|c|c|c|c|}
\hline Theme & $\mathrm{MOH}^{\mathrm{a}}(\mathrm{n}=271), \mathrm{n}(\%)$ & $\mathrm{CDC}^{\mathrm{b}}(\mathrm{n}=94), \mathrm{n}(\%)$ & $\operatorname{PHE}^{\mathrm{c}}(\mathrm{n}=45), \mathrm{n}(\%)$ \\
\hline Preventive measures & $60(22.1)$ & $50(53.2)$ & $18(40.0)$ \\
\hline Situation update & $78(28.8)$ & $21(22.3)$ & $7(15.6)$ \\
\hline Disease information & $16(5.9)$ & $17(18.1)$ & $17(37.8)$ \\
\hline Public reassurance & $32(11.8)$ & $2(2.1)$ & $0(0.0)$ \\
\hline Falsehood correction & $16(5.9)$ & $0(0.0)$ & $0(0.0)$ \\
\hline Others & $69(25.5)$ & $4(4.3)$ & $3(6.7)$ \\
\hline
\end{tabular}

aMOH: Ministry of Health.

${ }^{\mathrm{b}} \mathrm{CDC}$ : Centers for Disease Control and Prevention.

${ }^{\mathrm{c}}$ PHE: Public Health England.

\section{Sentiment and Emotion Analyses of Comments to COVID-19 Posts}

The number of CPP was highest for the CDC, which was attributed to the high number of followers on their Facebook page (Table 4). Although the $\mathrm{MOH}$ had fewer number of followers than PHE, the number of comments received for the MOH's COVID-19 posts were 5 times more than PHE. This observation can also be attributed to the high number of COVID-19 posts for the MOH vs PHE's COVID-19 posts. Interestingly, the mean CPP of PHE was still higher than the $\mathrm{MOH}$. The average SP scores of all three PHAs were close to the neutral sentiment mark of zero with only the MOH being slightly positive. Correspondingly, the PNSR and PNER of the $\mathrm{MOH}$ were much higher than the values for the CDC and PHE. However, since all these values were below 1 , it is an indication that there were more negative sentiments and emotions conveyed in the comments. The CDC and PHE received predominantly negative comments from their followers based on the low PNSR and PNER values.

In Figure 2, the emotion categories are plotted against the sentiment categories, with a darker shade of the box reflecting a higher number of comments. We observed that most of the comments to the $\mathrm{MOH}$ posts were angry $(n=1704,33.9 \%)$, with 1215 being angry and negative and 489 being angry and very negative. Anger is also the most prevalent emotion for the CDC $(n=12,634 / 42,470,29.8 \%)$ and PHE $(n=300 / 977,30.7 \%)$ posts. For the $\mathrm{MOH}$, the negative emotions (anger, disgust, fear, and sadness) account for $62.0 \%(\mathrm{n}=3119 / 5032)$ of the comments, while positive emotions (trust and joy) accounted for $33.0 \%$ $(\mathrm{n}=1655)$ of the comments. In the case of the CDC and PHE, negative emotions accounted for $63.0 \%(n=26,716 / 42,470)$ and $66.2 \%(\mathrm{n}=647 / 977)$ of the comments, respectively, and positive emotions accounted for $28.2 \%(n=11,987)$ and $26.0 \%(n=254)$ of comments, respectively. Since emotions have a direct effect on sentiments, the negative sentiments accounted for the majority of the comments $(n=2431 / 5032,48.3 \%$; $\mathrm{n}=21,015 / 42,470,49.5 \%$; and $\mathrm{n}=491 / 977,50.3 \%$ for the $\mathrm{MOH}$, the $\mathrm{CDC}$, and $\mathrm{PHE}$, respectively). The $\mathrm{MOH}$ had a higher percentage of positive sentiments $(n=1725 / 5032,34.3 \%)$ compared to the CDC $(n=12,256 / 42,470,28.9 \%)$ and PHE $(\mathrm{n}=277 / 977,28.4 \%)$.

The temporal trend analysis (Figure 3) provides more information compared to the snapshots provided in Table 4 and Figure 2. For instance, we observed that the number of comments increased significantly over time for the CDC posts, while the number of comments for the $\mathrm{MOH}$ posts appeared to have decreased over time. Among the three agencies, PHE had the highest degree of fluctuations in SP scores, with many negative and few positive spikes. The SP scores were mostly negative for the CDC posts, while SP scores were positive for the $\mathrm{MOH}$ on several occasions, which contributed to an average SP that tends toward neutral. The MOH had the highest number of days with positive sentiments, particularly the period between February 16, 2020, and March 5, 2020, which could be due to a relatively high number of appreciation posts $(n=9)$ during that period. 
Table 4. Emotion and sentiment analyses of COVID-19 Facebook comments.

\begin{tabular}{lllllll}
\hline Agency & COVID-19 $9^{\mathrm{a}}$ posts, $\mathrm{n}$ & Comments, $\mathrm{n}$ & $\mathrm{CPP}^{\mathrm{b}}$, mean $(\mathrm{SD})$ & $\mathrm{SP}^{\mathrm{c}}$, mean $(\mathrm{SD})$ & PNSR $^{\mathrm{d}}$, mean $(\mathrm{SD})$ & PNER $^{\mathrm{e}}$, mean $^{(\mathrm{SD})}$ \\
\hline $\mathrm{MOH}^{\mathrm{f}}$ & 271 & 5032 & $18.57(30.04)$ & $0.02(0.25)$ & $0.94(2.11)$ & $0.84(1.79)$ \\
$\mathrm{CDC}^{\mathrm{g}}$ & 94 & 42,470 & $451.81(529.09)$ & $-0.09(0.06)$ & $0.57(0.16)$ & $0.41(0.12)$ \\
PHE $^{\mathrm{h}}$ & 45 & 977 & $21.71(31.89)$ & $-0.14(0.26)$ & $0.55(0.49)$ & $0.44(0.54)$ \\
\hline
\end{tabular}

${ }^{\mathrm{a} C O V I D-19:}$ coronavirus disease.

${ }^{\mathrm{b}} \mathrm{CPP}$ : comments per post.

${ }^{\mathrm{c}} \mathrm{SP}$ : sentiment polarity score.

${ }^{\mathrm{d}}$ PNSR: positive to negative sentiments ratio.

${ }^{\mathrm{e}} \mathrm{PNER}$ : positive to negative emotions ratio.

${ }^{\mathrm{f}} \mathrm{MOH}$ : Ministry of Health.

${ }^{\mathrm{g}} \mathrm{CDC}$ : Centers for Disease Control and Prevention.

${ }^{h}$ PHE: Public Health England.

Figure 2. Sentiment and emotions heat map for coronavirus disease Facebook comments (left: Ministry of Health; middle: Centers for Disease Control and Prevention; right: Public Health England).
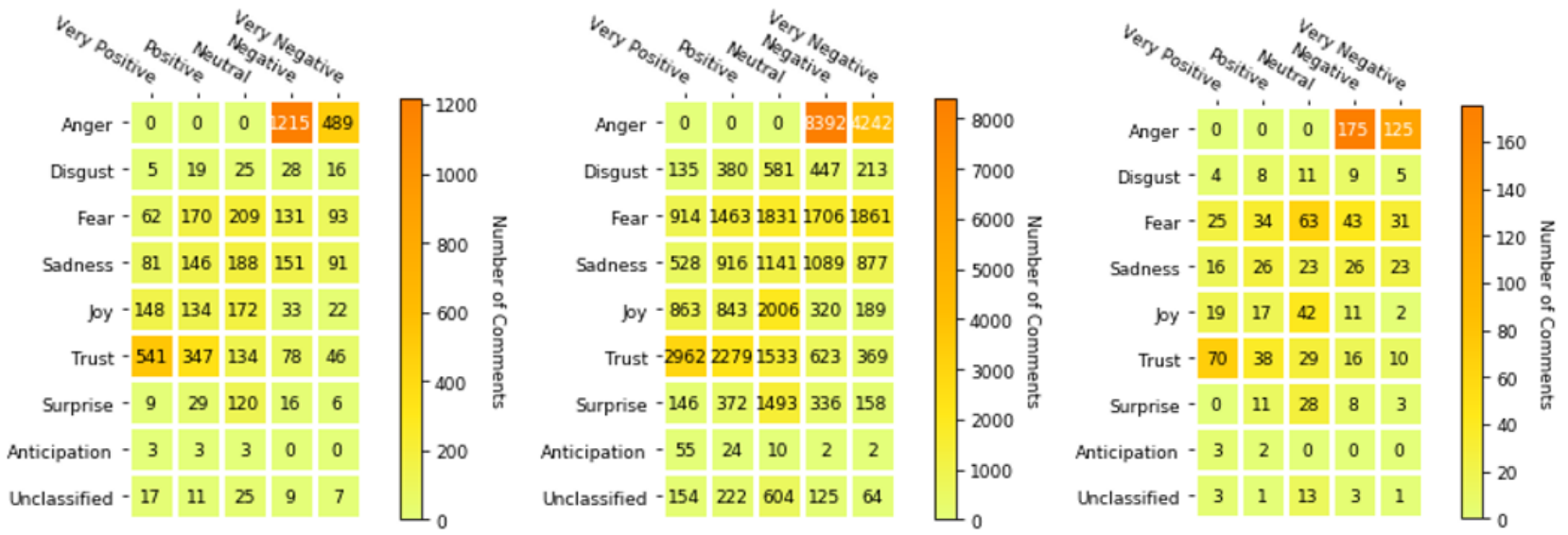

Figure 3. Temporal trend analysis for the number of comments and sentiment polarity. CDC_CC: Centers for Disease Control and Prevention's number of comments; CDC_SP: Centers for Disease Control and Prevention's sentiment polarity score; DORSCON: Disease Outbreak Response System Condition; $\mathrm{MOH} \_\mathrm{CC}$ : Ministry of Health's number of comments; MOH_SP: Ministry of Health's sentiment polarity score; PHE_CC: Pubilc Health England's number of comments; PHE_SP: Public Health England's sentiment polarity score; SG: Singapore; WHO: World Health Organization.

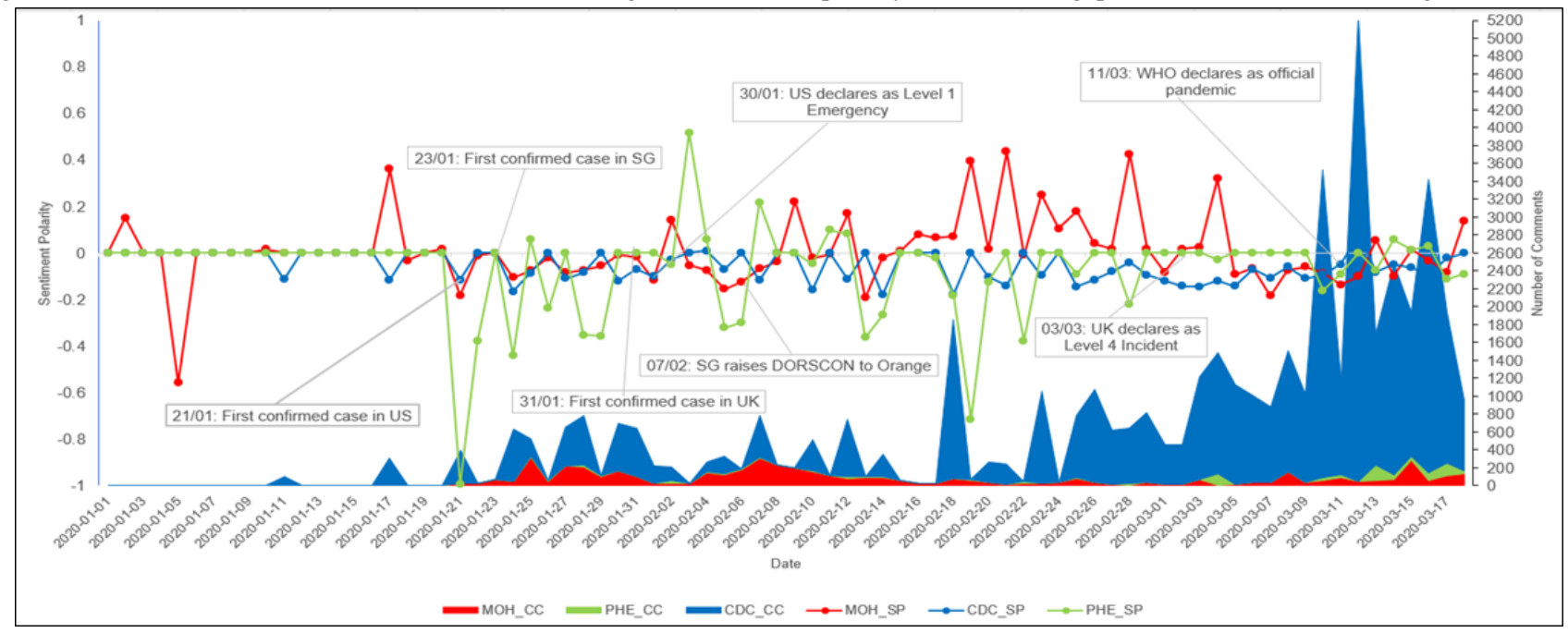




\section{Identification of Toxicity in Comments of COVID-19 Posts}

The CDC had the highest number of toxic comments, followed by the MOH and PHE (Table 5). PHE had the highest average of likes per toxic comment (LPTC) compared to the CDC and the MOH. For the PHE page, toxic comments received more likes and replies from its followers as compared to nontoxic comments. The mean LPTC for PHE was two times higher compared to nontoxic comments. Similarly, toxic comments received more replies compared to nontoxic comments.

Table 5. Summary of coronavirus disease toxic comments.

\begin{tabular}{lllllll}
\hline Agency & Total comments, $\mathrm{n}$ & Toxic comments, $\mathrm{n}(\%)$ & LPTC $^{\mathrm{a}}$, mean & LPNC $^{\mathrm{b}}$, mean & RPTC $^{\mathrm{c}}$ mean & RPNC $^{\mathrm{d}}$, mean $^{2}$ \\
\hline $\mathrm{MOH}^{\mathrm{e}}$ & 5032 & $58(0.01)$ & 0.47 & 1.38 & 0.14 & 0.43 \\
$\mathrm{CDC}^{\mathrm{f}}$ & 42,470 & $728(0.01)$ & 0.89 & 1.99 & 0.13 & 0.71 \\
$\mathrm{PHE}^{\mathrm{g}}$ & 977 & $12(0.01)$ & 2.42 & 1.44 & 0.92 & 0.60 \\
\hline
\end{tabular}

${ }^{\mathrm{a}}$ LPTC: likes per toxic comment.

${ }^{\mathrm{b}} \mathrm{LPNC}$ : likes per nontoxic comment.

${ }^{c}$ RPTC: replies per toxic comment.

${ }^{\mathrm{d}} \mathrm{RPNC}$ : replies per nontoxic comment.

${ }^{\mathrm{e}} \mathrm{MOH}$ : Ministry of Health.

${ }^{\mathrm{f}} \mathrm{CDC}$ : Centers for Disease Control and Prevention.

${ }^{g}$ PHE: Public Health England.

\section{Discussion}

\section{Principal Findings}

Among the three agencies, the $\mathrm{MOH}$ was the most active in using Facebook to reach out to its followers in terms of posting frequency, with an average of around 4 posts daily, exceeding both the CDC and PHE. The MOH displayed a similar active outreach strategy during the previous Zika outbreak in 2016 by ramping up engagement online with more frequent posting [21]. The MOH COVID-19 posts received more attention from their followers compared to the previous outbreak. For instance, the average number of comments received per post was 3.6 for Zika posts [21], while for COVID-19 posts, the average has increased to 15.6 .

The posting frequency of the CDC and PHE on matters pertaining to COVID-19 was low in the initial peri-COVID-19 phase; this was because a substantial number of posts were still dedicated to other public health topics (eg, mental health, food disease outbreak, chronic disease management). This may reflect that both countries perceived the risk to be low or possibly that the outbreak was still largely confined within Asia. At the time of analysis, the epicenter of the COVID-19 pandemic was Wuhan, China with several other Asian countries, including Singapore. We observed that the volume of updates on COVID-19 related to prevention from the CDC and PHE increased toward the end of the analysis period, which parallels the surge in case count in both the United States and England. Given the large number of followers on the CDC and PHE Facebook pages, it is a missed opportunity that the CDC and PHE did not engage with their followers more intensively using Facebook. We observed that Facebook users who engaged with the CDC or PHE posts were more likely to share rather than to react or comment on the posts. Hence, Facebook may possibly be a useful platform for the CDC or PHE to disseminate information for Facebook users to propagate to others.
In our analysis of the PHAs' post content, we restricted the number of themes to six to focus on major themes, unlike earlier studies where more themes were used with a meagre number of posts for certain themes [26]. The posts from the MOH were more diverse with frequent updates on preventive measures, travel advisories, disease information, falsehood correction, and even appreciation for health care workers and other frontline staff. This was in contrast to the posts from the CDC and PHE, where the messages were mostly focused on preventive measures and sporadic situation updates. Falsehood corrections are in need during this pandemic, as an earlier study identified that misleading Facebook posts acquired more popularity than accurate posts during the Zika outbreak in the United States [43]. On this point, we observed that the MOH has adopted misinformation debunking as one of its community and social measures for handling the COVID-19 situation [6]. The current distribution of themes may reflect the different phases that the three countries were going through during the time of our analyses. We anticipate that as the pandemic develops across each country the themes of the posts will continually evolve. Nevertheless, there is a need to enhance awareness and not undermine the possibility of a serious outbreak during the precrisis period [22]. From our analyses, we did not identify much evidence to show attempts at such efforts from the CDC and PHE in their official Facebook pages.

In previous disease pandemics, negative sentiments were generally prevalent in social media [23]. We made similar findings in our analyses where the majority of the posts conveyed anger emotions and negative sentiments. For the $\mathrm{MOH}$, however, we noticed that over time (from mid-February to the first week of March 2020), Facebook users began to be more positive about the government's response to the pandemic. This demonstrates that monitoring sentiments and emotions on social media can help PHAs gauge the effectiveness of their public health education efforts on Facebook. Another observation that supports the monitoring of sentiments and 
emotions on social media is that the number of comments tend to spike in conjunction with specific events. For example, our data showed that the number of comments sharply rose in association with the first confirmed case in the United States (January 21, 2020), when Singapore rose its DORSCON level to orange (February 7, 2020), when the United Kingdom declared COVID-19 as a Level 4 incident (March 3, 2020), and when the WHO declared COVID-19 a pandemic (March 11, 2020).

The prevalence of toxic comments for all three Facebook pages' COVID-19 posts was fairly low. It is possible that the majority of the toxic comments had been removed (for instance, the CDC has a policy that profane and obscene comments can be deleted [44]), and what was analyzed were those that were not filtered. The US President has repeatedly referred to SARS coronavirus 2 as the "Chinese virus," and this may have led to anti-Asian sentiments [45]. This may explain why the CDC had the highest number of toxic comments. Fortunately, the volume of such comments remained low, and the agreement with such toxic comments was also low as reflected by the low average number of likes. PHAs should consider dedicating resources during a pandemic to manage toxic comments as well as combat falsehood. We observed that both the CDC and PHE did not have any post to correct falsehoods, unlike the MOH. In contrast, social media platforms have been proactive in setting up centralized hubs dedicated to COVID-19 updates and information such as the COVID-19 Information Center on Facebook [46] or the COVID-19 Information and Resources collection on Google [47] to direct social media users to trusted and reliable information.

\section{Limitations}

In this study, we analyzed data from Facebook only. However, PHAs may have used other social media platforms such as Twitter and YouTube to disseminate public health information to their citizens. Thus, this study's findings may not fully represent the overall social media outreach efforts of PHAs during the COVID-19 pandemic. In addition, the sentiments captured on Facebook comments may not reflect the users of other social media platforms, as the user profiles of these various platforms are known to be different. Furthermore, PHAs may currently still use traditional news and mass media channels to reach the public with information, updates, and guidance measures. Hence, PHAs' outreach efforts in social media platforms is supplementary, and these platforms are considered either as a resource for additional information or for reaching out to people who no longer follow traditional news and mass media channels. Another limitation of the study was that we have limited our analyses of the Facebook followers' response to posts initiated by the PHAs. We have not, for instance, analyzed the comments within private circles or closed groups that may be different in nature compared to publicly disclosed comments. Our paper has focused on the comparative analysis of how three PHAs have used Facebook for COVID-19 communications strategy. We did not evaluate if the PHAs' use of social media has improved over time as they gain experience from dealing with other infectious diseases such as the H1N1 swine flu pandemic, Ebola epidemic, or Zika outbreak. As social media platforms become more prominent, its users and the interactions among its users evolve. Optimal use of these platforms for public health communications will benefit from constant reflection and critical appraisal of what strategies have worked and what have not. Finally, the thematic analysis may be more robust if independent coding of the posts was conducted. However, the huge number of posts rendered this process time-consuming. Accordingly, the review and confirmation of the themes from a second coder was sought as an acceptable compromise.

\section{Conclusions}

The Facebook postings by the PHAs in this study provided some insights into their governments' COVID-19 broader communication strategy. Through our study, we identified differences in the Facebook-based outreach and engagement efforts of three developed countries during the prepandemic and peripandemic periods of COVID-19. The differences were found in terms of both posting frequency and themes in posts. The change in sentiments in response to specific outreach events were also observed. On the whole, the $\mathrm{MOH}$ stepped up its outreach efforts on Facebook more intensively compared to the CDC and PHE. We hope that our findings will be of interest to PHAs and health science researchers who study pandemics in the context of social media. In our upcoming work, we intend to conduct studies in two related directions. In our first set of studies, we intend to collect more data from the same three PHA Facebook pages and analyze the results with data segregated in three phases (pre-COVID-19, peri-COVID-19, and post-COVID-19). In the second set of studies, we intend to analyze the outreach efforts of other countries during this COVID-19 pandemic to understand the effectiveness and shortfalls of strategies used by different countries.

\section{Acknowledgments}

We would like to thank Miss Yu-Ting, CHEN (research assistant) for providing administrative support to submitting this manuscript. ASR was hired under a grant from the National University Health System Strategic Funds titled "Phen-Gen Research Repository and Pre-Emptive Pharmacogenomics." During COVID-19, safe distancing measures prevented him from accessing data stored in a dedicated workstation without internet access. He was thus reassigned to this study. The grantor had no role in the design and conduct of the study; collection, management, analysis, and interpretation of the data; preparation, review, or approval of the manuscript; or the decision to submit the manuscript for publication. This work was also supported by the Centre for Health Services and Policy Research, a center under the National University Health Systems Pte Ltd and Saw Swee Hock School of Public Health, National University of Singapore. We would like to thank the Perspective API team of Google for generously extending the usage quota of their API service, which we used to measure the toxicity of Facebook comments for our research study. 


\section{Authors' Contributions}

H-LW conceptualized the study, interpreted the data, critically revised the manuscript for important intellectual content, and provided supervision. ASR designed the study; acquired, analyzed, and interpreted the data; drafted the manuscript; and critically revised the manuscript for important intellectual content. SGT analyzed and interpreted the data and critically revised the manuscript for important intellectual content. All authors approved the final version of the manuscript.

\section{Conflicts of Interest}

None declared.

\section{Multimedia Appendix 1}

Emojis and the associated emotions.

[DOCX File, 16 KB-Multimedia Appendix 1]

\section{References}

1. Worldometer. 2020. Countries where COVID-19 has spread URL: https://www.worldometers.info/coronavirus/ countries-where-coronavirus-has-spread/ [accessed 2020-04-11]

2. Uras U. Al Jazeera. 2020 Apr 08. Coronavirus: comparing COVID-19, SARS and MERS URL: https://www.aljazeera.com/ news/2020/04/coronavirus-comparing-covid-19-sars-mers-200406165555715.html [accessed 2020-04-11]

3. Holshue ML, DeBolt C, Lindquist S, Lofy KH, Wiesman J, Bruce H, et al. First case of 2019 novel coronavirus in the United States. N Engl J Med 2020 Mar 05;382(10):929-936. [doi: 10.1056/nejmoa2001191]

4. Moss P, Barlow G, Easom N, Lillie P, Samson A. Lessons for managing high-consequence infections from first COVID-19 cases in the UK. Lancet 2020 Mar 14;395(10227):e46 [FREE Full text] [doi: 10.1016/S0140-6736(20)30463-3] [Medline: 32113507]

5. Abdullah Z, Salamat H. Channel News Asia. 2020 Jan 23. Singapore confirms first case of Wuhan virus URL: https://www. channelnewsasia.com/news/singapore/wuhan-virus-pneumonia-singapore-confirms-first-case-12312860 [accessed 2020-04-11]

6. Lee V, Chiew C, Khong W. Interrupting transmission of COVID-19: lessons from containment efforts in Singapore. J Travel Med 2020 Mar 13:e [FREE Full text] [doi: 10.1093/jtm/taaa039] [Medline: 32167146]

7. Ministry of Health. 2020 Feb 07. Risk assessment raised to DORSCON orange URL: https://www.moh.gov.sg/ news-highlights/details/risk-assessment-raised-to-dorscon-orange [accessed 2020-04-11]

8. World Health Organization. 2020 Mar 11. WHO Director-General's opening remarks at the media briefing on COVID-19 - 11 March 2020 URL: https://www.who.int/dg/speeches/detail/

who-director-general-s-opening-remarks-at-the-media-briefing-on-covid-19---11-march-2020 [accessed 2020-04-11]

9. Barron L. Time. 2020 Mar 13. What we can learn from Singapore, Taiwan and Hong Kong about handling coronavirus URL: https://time.com/5802293/coronavirus-covid19-singapore-hong-kong-taiwan/ [accessed 2020-04-10]

10. Teo J. The Straits Times. 2020 Mar 10. Coronavirus: WHO praises Singapore's containment of Covid-19 outbreak URL: https://www.straitstimes.com/singapore/health/coronavirus-who-praises-singapores-containment-of-covid-19-outbreak [accessed 2020-04-09]

11. The Straits Times. 2020 Mar 13. Covid-19 situation is serious but under control in Singapore: PM Lee URL: https://www. straitstimes.com/singapore/covid-19-situation-is-serious-but-under-control-in-spore-pm-lee [accessed 2020-04-09]

12. Facebook. Facebook. 2020 Mar 31. Coronavirus: lessons learned from Singapore URL: https://www.facebook.com/ nus.sshsph/posts/2766847946771712? tn =-R [accessed 2020-04-09]

13. Facebook. 2020 Apr 08. COVID-19: lessons learned? What comes next? URL: https://www.facebook.com/nus.sshsph/ posts/2784851854971321 [accessed 2020-04-09]

14. Costello A. The Guardian. 2020 Mar 15. The UK's Covid-19 strategy dangerously leaves too many questions unanswered URL: https://www.theguardian.com/commentisfree/2020/mar/15/ uk-covid-19-strategy-questions-unanswered-coronavirus-outbreak [accessed 2020-04-10]

15. Biesecker M. Global News. 2020 Apr 05. U.S. 'wasted' months before preparing for COVID-19 pandemic: former health secretary URL: https://globalnews.ca/news/6780562/coronavirus-covid-wasted-months/ [accessed 2020-04-10]

16. Hoffman SJ, Silverberg SL. Delays in global disease outbreak responses: lessons from H1N1, Ebola, and Zika. Am J Public Health 2018 Mar;108(3):329-333. [doi: 10.2105/AJPH.2017.304245] [Medline: 29345996]

17. Holmes BJ. Communicating about emerging infectious disease: the importance of research. Health Risk Soc 2008 Aug;10(4):349-360. [doi: 10.1080/13698570802166431]

18. Vaughan E, Tinker T. Effective health risk communication about pandemic influenza for vulnerable populations. Am $\mathbf{J}$ Public Health 2009 Oct;99(S2):S324-S332. [doi: 10.2105/ajph.2009.162537]

19. Wong JEL, Leo YS, Tan CC. COVID-19 in Singapore-current experience: critical global issues that require attention and action. JAMA 2020 Feb 20:e. [doi: 10.1001/jama.2020.2467] [Medline: 32077901]

20. Vijaykumar S, Jin Y, Nowak G. Social media and the virality of risk: the risk amplification through media spread (RAMS) model. J Homeland Secur Emerg Manage 2015:653. [doi: 10.1515/jhsem-2014-0072] 
21. Vijaykumar S, Meurzec R, Jayasundar K, Pagliari C, Fernandopulle Y. What's buzzing on your feed? Health authorities' use of Facebook to combat Zika in Singapore. J Am Med Informatics Assoc 2017;24(6):1159. [doi: 10.1093/jamia/ocx028]

22. Lwin M, Lu J, Sheldenkar A, Schulz P. Strategic uses of Facebook in Zika outbreak communication: implications for the crisis and emergency risk communication model. Int J Environ Res Public Health 2018 Sep 10;15(9):1974. [doi: 10.3390/ijerph15091974] [Medline: 30201929]

23. Mamidi R, Miller M, Banerjee T, Romine W, Sheth A. Identifying key topics bearing negative sentiment on Twitter: insights concerning the 2015-2016 Zika epidemic. JMIR Public Health Surveill 2019 Jun 04;5(2):e11036. [doi: 10.2196/11036] [Medline: 31165711$]$

24. de Araujo DHM, de Carvalho EA, da Motta CLR, da Silva Borges MR, Gomes JO, de Carvalho PVR. Social networks applied to Zika and H1N1 epidemics: a systematic review. Springer, Cham 2019:679. [doi: 10.1007/978-3-319-96089-0_74]

25. Strekalova YA. Health risk information engagement and amplification on social media. Health Educ Behav 2017 Apr;44(2):332-339. [doi: 10.1177/1090198116660310] [Medline: 27413028]

26. Vijaykumar S, Nowak G, Himelboim I, Jin Y. Virtual Zika transmission after the first U.S. case: who said what and how it spread on Twitter. Am J Infect Control 2018 May;46(5):549-557. [doi: 10.1016/j.ajic.2017.10.015] [Medline: 29306490]

27. World Health Organization. 2018 Jan 10. Communicating risk in public health emergencies: a WHO guideline for emergency risk communication (ERC) policy and practice URL: https://www.who.int/risk-communication/guidance/download/en/ [accessed 2020-04-09]

28. Glik DC. Risk communication for public health emergencies. Annu Rev Public Health 2007;28:33-54. [doi: 10.1146/annurev.publhealth.28.021406.144123] [Medline: 17222081$]$

29. Jünger J, Keyling T. GitHub. 2019. Facepager URL: https://github.com/strohne/Facepager/ [accessed 2020-04-06]

30. Facebook. Ministry of Health, Singapore URL: https://www.facebook.com/sghealthministry [accessed 2020-05-08]

31. Facebook. CDC URL: https://www.facebook.com/CDC [accessed 2020-05-08]

32. Facebook. Public Health England URL: https://www.facebook.com/PublicHealthEngland/ [accessed 2020-05-08]

33. Ministry of Health. 2020 Jan 02. Precautionary measures in response to severe pneumonia cases in Wuhan, China URL: https://www.moh.gov.sg/news-highlights/details/ precautionary-measures-in-response-to-severe-pneumonia-cases-in-wuhan-china [accessed 2020-04-10]

34. Erlingsson C, Brysiewicz P. A hands-on guide to doing content analysis. Afr J Emerg Med 2017 Sep;7(3):93-99. [doi: 10.1016/J.AFJEM.2017.08.001]

35. Hutto C, Gilbert E. VADER: a parsimonious rule-based model for sentiment analysis of social media text. AAI Publications, Eighth International AAAI Conference on Weblogs and Social Media 2014:A [FREE Full text]

36. Plutchik R. Emotions: a general psychoevolutionary theory. In: Approaches to Emotion. London: Psychology Press; May 22, 2014:A.

37. Felbo B, Mislove A, Søgaard A, Rahwan I, Lehmann S. Using millions of emoji occurrences to learn any-domain representations for detecting sentiment, emotion and sarcasm. 2017 Presented at: 2017 Conference on Empirical Methods in Natural Language Processing; September 2017; Copenhagen, Denmark. [doi: 10.18653/v1/d17-1169]

38. Evers C, Adriaanse M, de Ridder DT, de Witt Huberts JC. Good mood food. Positive emotion as a neglected trigger for food intake. Appetite 2013 Sep;68:1-7. [doi: 10.1016/j.appet.2013.04.007] [Medline: 23602962]

39. Al-Hajjar D, Syed A. Applying sentiment and emotion analysis on brand tweets for digital marketing. 2015 Dec 21 Presented at: 2015 IEEE Jordan Conference on Applied Electrical Engineering and Computing Technologies (AEECT); 3-5 Nov 2015; Amman, Jordan. [doi: 10.1109/aeect.2015.7360592]

40. Daniulaityte R, Lamy F, Smith G, Nahhas R, Carlson R, Thirunarayan K, et al. "Retweet to pass the blunt": analyzing geographic and content features of cannabis-related tweeting across the United States. J Stud Alcohol Drugs 2017 Nov;78(6):910-915 [FREE Full text] [doi: 10.15288/jsad.2017.78.910] [Medline: 29087826]

41. Cheng J, Bernstein M, Danescu-Niculescu-Mizil C, Leskovec J. Anyone can become a troll: causes of trolling behavior in online discussions. 2017 Presented at: 2017 ACM Conference on Computer Supported Cooperative Work and Social Computing; 2017; New York, NY p. 1217-1230. [doi: 10.1145/2998181.2998213]

42. Google. Perspective API URL: https://www.perspectiveapi.com/\#/home [accessed 2020-04-06]

43. Sharma M, Yadav K, Yadav N, Ferdinand KC. Zika virus pandemic-analysis of Facebook as a social media health information platform. Am J Infect Control 2017 Mar 01;45(3):301-302. [doi: 10.1016/j.ajic.2016.08.022] [Medline: 27776823]

44. CDC. Social media public comment policy URL: https://www.cdc.gov/socialmedia/tools/CommentPolicy.html [accessed 2020-05-08]

45. The Washington Post. 2020. Trump calling coronavirus "Chinese virus" encourages racism against Asian Americans, experts say URL: https://www.washingtonpost.com/nation/2020/03/20/coronavirus-trump-chinese-virus/ [accessed 2020-04-09]

46. Facebook. Coronavirus (COVID-19) Information Centre URL: https://www.facebook.com/coronavirus_info/ [accessed 2020-04-10]

47. Google. COVID-19 information \& resources URL: https://www.google.com/covid19/ [accessed 2020-04-13] 


\title{
Abbreviations
}

API: application program interface

CDC: Centers for Disease Control and Prevention

COVID-19: coronavirus disease

CPP: comments per post

DORSCON: Disease Outbreak Response System Condition

LPTC: likes per toxic comment

MOH: Ministry of Health

PHA: public health authority

PHE: Public Health England

PNER: positive to negative emotions ratio

PNSR: positive to negative sentiment ratio

PPD: posts per day

RPP: reactions per post

SARS: severe acute respiratory syndrome

SP: sentiment polarity

SPP: shares per post

WHO: World Health Organization

\author{
Edited by G Eysenbach; submitted 13.04.20; peer-reviewed by G Nowak, A Dormanesh, A Natale, C Mather; comments to author \\ 06.05.20; revised version received 11.05.20; accepted 12.05.20; published 19.05.20 \\ Please cite as: \\ Sesagiri Raamkumar A, Tan SG, Wee HL \\ Measuring the Outreach Efforts of Public Health Authorities and the Public Response on Facebook During the COVID-19 Pandemic \\ in Early 2020: Cross-Country Comparison \\ J Med Internet Res 2020;22(5):e19334 \\ URL: http://www.jmir.org/2020/5/e19334/ \\ doi: $10.2196 / 19334$ \\ PMID: 32401219
}

(C)Aravind Sesagiri Raamkumar, Soon Guan Tan, Hwee Lin Wee. Originally published in the Journal of Medical Internet Research (http://www.jmir.org), 19.05.2020. This is an open-access article distributed under the terms of the Creative Commons Attribution License (https://creativecommons.org/licenses/by/4.0/), which permits unrestricted use, distribution, and reproduction in any medium, provided the original work, first published in the Journal of Medical Internet Research, is properly cited. The complete bibliographic information, a link to the original publication on http://www.jmir.org/, as well as this copyright and license information must be included 